\section{Comment on "Determination of the Chemical Potential of Polymeric Systems from Monte Carlo Simulations"}

In a recent Letter, Kumar, Szleifer, and Panagiotopoulos [1] reported a novel method to determine the chemical potential of a polymer and applied this method to a system containing chains of twenty segments. In Ref. [1], a central role is played by the "incremental chemical potential," i.e., the derivative of the chemical potential with respect to chain length. The chemical potential of the entire chain can be estimated from the incremental chemical potential under the assumption that the latter is independent of chain length. In order to show that this assumption is reasonable, Kumar, Szleifer, and Panagiotopoulos report data which suggest that the incremental chemical potential of an isolated chain is indeed effectively constant for all but the shortest chains. Recently, we have developed a rigorous scheme to compute the chemical potential of arbitrary chain molecules [2]. In this Comment, we use the latter method to compute both the total and the incremental chemical potential of an isolated chain at $T=2.0 \epsilon / k_{B}$. We find that the incremental chemical potential is not constant.

In Ref. [2] we have shown that one can calculate directly the nonbonded contribution to the excess chemical potential of a flexible chain molecule

$$
\beta \mu^{\mathrm{ex}}(v) \equiv \beta\left[\mu_{\text {chain }}(v)-(v-1) \mathfrak{F}(\beta)\right],
$$

where $\beta=1 / k_{B} T, \mu_{\text {chain }}(v)$ is the chemical potential of a chain with $v$ beads, and $(v-1) \mathcal{F}(\beta)$ is the chemical potential of a chain with only bonded interactions, for which the chemical potential can be calculated analytically for the model in Ref. [1]. In the spirit of Kumar, Szleifer, and Panagiotopoulos we define the excess incremental chemical potential as

$$
\beta \mu_{\mathrm{r}}^{\mathrm{ex}}(v) \equiv \beta\left[\mu^{\mathrm{ex}}(v+1)-\mu^{\mathrm{ex}}(v)\right] .
$$

Figure 1 shows that the nonbonded contribution is clearly not constant for the chains studied in Ref. [1]. This finding is of some relevance, as the assumption of constant incremental chemical potential was actually used in [1] to estimate a liquid-vapor coexistence point for this chain. Our conclusion concerning the length dependence of the incremental chemical potential differs from that of Kumar, Szleifer, and Panagiotopoulos because these authors did not subtract the contribution to $\mu_{\mathrm{r}}(v)$ due to bonded interactions. This contribution is by construction independent of chain length. Unfortunately this "trivial" term-in the sense that it is irrelevant for phase behavior - is 1-2 orders of magnitude larger than the excess chemical potential. In fact, the statistical noise in the simulation of Kumar, Szleifer, and Panagiotopoulos is larger than the total nonbonded contributions. As a consequence, the constant contribution to the excess chemical potential obscures any meaningful length

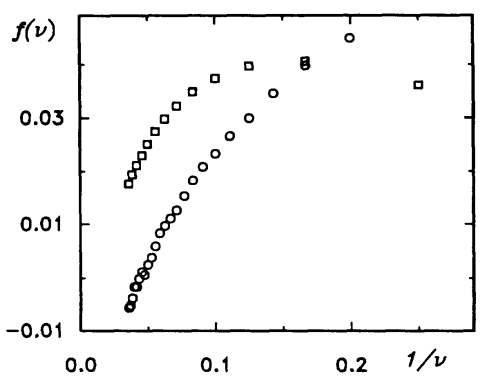

FIG. 1. The chemical potential as a function of the reciprocal chain length $1 / v$. The incremental chemical potential is represented by $\circ, f(v) \equiv \beta \mu_{\mathrm{r}}^{\text {ex }}(v)$. The chemical potential of the entire chain per bead, for which $f(v) \equiv \beta \mu^{\text {ex }}(v) / v$, is given by $\square$.

\section{dependence.}

We have also tested the assumption that the total nonbonded chemical potential of an isolated chain of length $v$ can be obtained from the incremental chemical potential and a direct calculation of the total chemical potential of a "short" chain of length $v_{0}$. In order for this approach to work, $\mu_{\text {chain }}(v) / v$ should become linear in $1 / v$ for $v$ larger than the "crossover length" $v_{0}$. The data in Fig. 1 indicate that, if there is a linear regime at all, it only starts at a value of $v_{0}$ that is comparable to the total length of the chains studied in Ref. [1]. We wish to stress, however, that we agree with Ref. [1] that the incremental chemical potential is, in principle, a very useful quantity to estimate the chemical potential of "sufficiently" long chain molecules. What is "sufficiently long" will depend on the system under study [2]. Clearly, a better understanding of the behavior of the crossover length is highly desirable.

The authors thank S. K. Kumar, I. Szleifer, and A. Z. Panagiotopoulos for valuable comments. The work of the FOM Institute is part of the research program of the Stichting Fundamenteel Onderzoek der Materie.

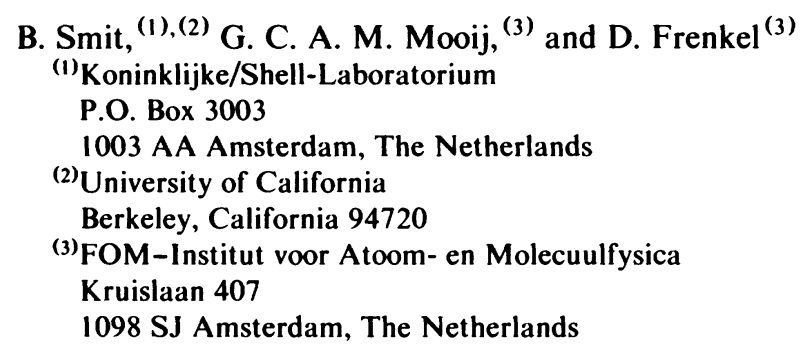

Received 14 February 1992

PACS numbers: 82.20.Wt, 05.70.-a, 64.70.Fx, 82.60.Lf

[1] S. K. Kumar, I. Szleifer, and A. Z. Panagiotopoulos, Phys. Rev. Lett. 66, 2935 (1991).

[2] D. Frenkel and B. Smit, Mol. Phys. 75, 983 (1992); D. Frenkel et al., J. Phys. Condens. Matter 4, 3053 (1992). 